\title{
In-vivo, cardiac-cycle related intimal displacement of coronary plaques assessed by 3-D ECG-gated intravascular ultrasound: exploring its correlate with tissue deformability identified by palpography
}

\author{
Gastón A. Rodriguez-Granillo, Pierfrancesco Agostoni, Héctor M. García-García, \\ Pim de Feyter \& Patrick W. Serruys \\ Erasmus Medical Center, Thoraxcenter, Rotterdam, The Netherlands
}

Received 20 May 2005; accepted in revised form 7 June 2005

Key words: atherosclerosis, imaging, mechanical strain, ultrasonography

\begin{abstract}
Background: ECG-gated image acquisition of intravascular ultrasound (IVUS) has been shown to provide more accurate measurements at different phases of the cardiac cycle. Objective: We sought to explore the ability dynamic assessment of ECG-gated 3-D IVUS to identify deformable regions of coronary plaques, by testing the hypothesis that at a given pressure and region, a faster displacement of the intima would correspond to high strain (soft tissue) regions assessed by palpography. Methods: ECG-gated 3-D IVUS and palpograms were acquired using 30 and $20 \mathrm{MHz}$ IVUS imaging catheters respectively. Frames with high and/or low strain spots identified by palpography were randomly selected and the spots were assigned to a respective quadrant within the cross section. A color-blinded side-by-side view was performed to enable the co-localization of the same region. Subsequently, the pressure driven displacement of the intima was established for each quadrant and a binary score (significant displacement or no displacement) was decided. Results: One hundred and twenty-four quadrants were studied and the prevalence of highly deformable quadrants was low $(n=7,5.6 \%$ of the total). The sensitivity, specificity, positive predictive value and negative predictive value of 3-D ECG-gated IVUS to detect deformable quadrants as assessed by palpography were $42.9,87.2,16.7$, and $96.2 \%$ respectively. Conclusion: In this pilot in vivo study, the intimal displacement velocity in the radial direction assessed by gray-scale 3-D ECG-gated IVUS failed to correlate with highly deformable regions. However, these preliminary findings suggest that the absence of significant displacement of the intima might be accurate to predict the absence of deformable tissue.
\end{abstract}

\section{Introduction}

Despite major improvements in the management and diagnosis of patients with coronary artery disease, a large number of victims who are apparently healthy die suddenly without prior symptom $[1,2]$. Intensive efforts are currently been made to detect in vivo vulnerability features of coronary atherosclerotic plaques. Several catheter-based techniques have been developed with the aim of characterizing and eventually evaluating the effect of conventional and novel therapeutic intervention of such non-flow-limiting lesions [3-5].

An important patho-morphologic feature of vulnerable plaques is the eccentric accumulation of a lipid-rich necrotic core within the vessel wall, separated from the lumen by a thin fibrous cap. This observation led to the hypothesis that vulnerable 
lesions might have mechanical properties that differ from those of chronic stable lesions. Indeed, both plaque rupture and increased inflammatory markers have been reported to occur more frequently in regions with increased mechanical stress $[3,6,7]$.

Intravascular Ultrasound (IVUS) is an invasive diagnostic tool that provides a real-time, highresolution, tomographic view of coronary arteries. It thereby enables the assessment of morphology, severity and extension of coronary plaque. By reducing motion artifacts caused by the displacement of the catheter relative to the vessel wall during a pullback, ECG-gated image acquisition of IVUS has been shown to provide more accurate measurements with lower intra and interobserver variability [8-10]. In addition, it allows measurements at different phases of the cardiac cycle [8].

A recent study established that the luminal, pressure driven displacement of low echogenic (soft) plaques is faster than the one present in calcified lesions [11].

In this study, we sought to explore the ability of ECG-gated 3-D IVUS to identify deformable regions of coronary plaques, by testing the hypothesis that at a given pressure and region, a faster displacement of the intima would correspond to high strain regions as assessed by palpography, which represent soft (highly deformable) tissue.

\section{Methods}

Patients were eligible if they had a de novo, nonsignificant (angiographically $<50 \%$ ) stenosis in a native coronary artery. Patients were excluded from the study if any of the following conditions were present: (1) severe vessel tortuosity (2) severely calcified vessels. Written informed consent was obtained from all patients.

\section{Intravascular ultrasound acquisition}

IVUS was performed after intracoronary administration of nitrates using a single-element, $30 \mathrm{MHz}$ rotating transducer $\left(3.2 \mathrm{~F}\right.$ Ultracross $^{\mathrm{TM}}$, Boston Scientific Corp.). Cine runs, before and during contrast injection, were performed to define the position of the IVUS catheter $\geqslant 10 \mathrm{~mm}$ distal to a clear anatomical landmark. The ECG-gated image acquisition and digitization were performed by a 3-D image acquisition workstation (EchoScan, TomTec, Munich, Germany), which received the video signal input form the IVUS console and the ECG-signal from the patient. This system steered the ECG-gated stepping pullback device to withdraw the imaging transducer. The workstation considered the heart rate variability and only acquired images from cycles meeting a predetermined range. Premature beats were rejected.

If an $\mathrm{R}-\mathrm{R}$ interval failed to meet the preset range, the catheter remained at the same site until a cardiac cycle met the predetermined $\mathrm{R}-\mathrm{R}$ range. Subsequently, the transducer was withdrawn $0.2 \mathrm{~mm}$ and images were recorded. Image acquisition required on average $1 \mathrm{~min}$ per $\mathrm{cm}$.

\section{Palpography acquisition}

Palpograms were acquired using a $20-\mathrm{MHz}$ phased-array IVUS catheter (Volcano Therapeutics, Rancho Cordova, USA). Cine runs, before and during contrast injection, were performed to define the position of the IVUS catheter $\geqslant 10 \mathrm{~mm}$ distal to the same landmark used for the $30 \mathrm{MHz}$ catheter. Digital radiofrequency data were acquired using a custom-designed workstation.

Intravascular ultrasound palpography is a technique that allows the assessment of local mechanical tissue properties [3, 12]. At a defined pressure, soft tissue (lipid-rich) components will deform more than hard tissue components (fibrous-calcified) [13]. In coronaries, the tissue of interest is the vessel wall, whereas the blood pressure with its physiologic, systolic and diastolic changes during the heart cycle is used as the excitation force. Images obtained at different pressure levels are compared to determine the local tissue deformation.

Each palpogram represents the strain information for a certain cross section over the full cardiac cycle. The longitudinal resolution of the acquisitions depends on heart rate and pullback speed. With a heart rate of $60 \mathrm{bpm}$ and a pullback speed of $1.0 \mathrm{~mm} / \mathrm{s}$, the longitudinal resolution is $1.0 \mathrm{~mm}$. For palpography, catheter displacement is the main 

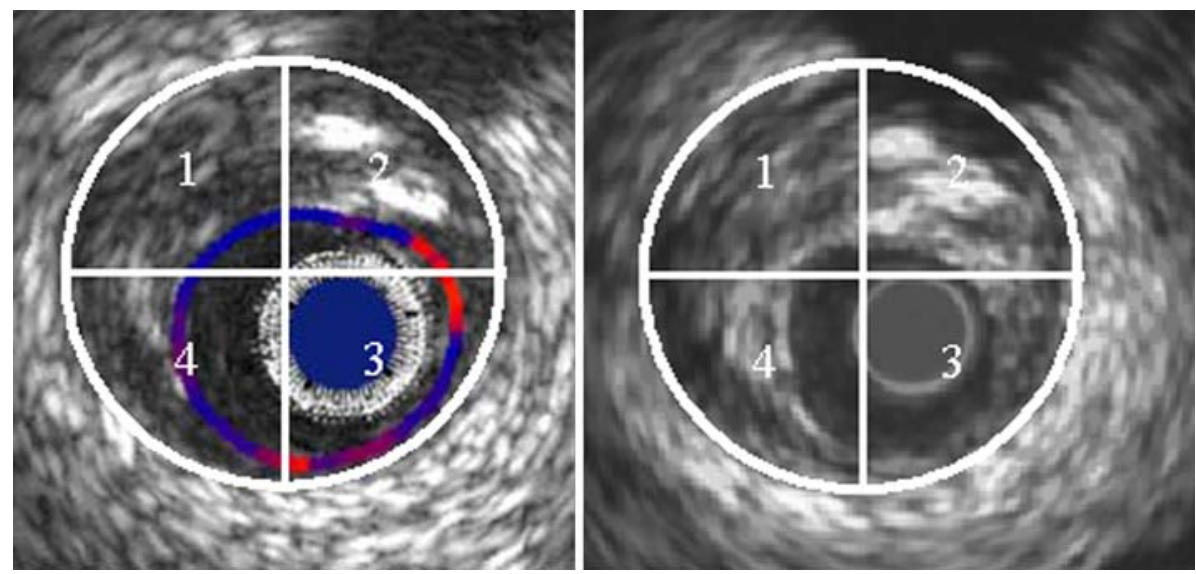

Figure 1. Matched cross-section imaged with palpography (left) and 3-D ECG-gated (right) intravascular ultrasound. A highly deformable plaque and significant intimal radial displacement is present in quadrant 3.

source of signal decorrelation and thus a source of error in strain estimation [14]. During the recordings, data were continuously acquired at a pullback speed of $1.0 \mathrm{~mm} / \mathrm{s}$ using a mechanical pullback device (Track Back II, Volcano Therapeutics) with simultaneous recording of the ECG and the aortic pressure. The data set is subdivided into heart cycles by use of the R wave of the ECG signal.

\section{Palpography analysis}

The local strain was calculated from the gated radiofrequency traces using cross-correlation analysis and displayed, color-coded, from blue (for $0 \%$ strain) through yellow (for $2 \%$ strain) via red. This color-coded circumferential image was superimposed on the cross-sectional IVUS image (Figure 1).

A region was defined as a high-strain spot when it had high strain $(\geqslant 0.9 \%$ at $4 \mathrm{~mm} \mathrm{Hg}$ pressure difference) that spanned an arc of at least $12^{\circ}$ at the surface of a plaque (identified on the IVUS recording) adjacent to low-strain regions $(<0.5 \%$ at $4 \mathrm{~mm} \mathrm{Hg}$ pressure difference). The highest value of strain was taken as the strain level of the spot. An independent experienced analyst randomly selected frames with high and/or low strain spots within the pullback analysis and positioned the spots in a respective quadrant according to the spatial location within the cross section (Figure 1).

\section{Qualitative IVUS analysis}

A color-blinded side-by-side view (gray-scale IVUS and Palpography) was undertaken to facilitate the identification of the same region with both techniques. Using longitudinal as well as cross-sectional views and with the aid of anatomical landmarks such as side-branches, veins, calcified spots and pericardium, three experienced IVUS analysts blinded for the palpography results identified the given frames. Images were rotated with the purpose of matching the orientation of the images provided in the palpogram. With the aid of dynamic evaluation of both longitudinal and cross-sectional views, the longitudinal movement of the catheter at the current frame was estimated qualitatively.

Subsequently, and provided that the longitudinal movement was not significant (determined by cyclic entrance/exit of identifiable anatomical landmarks), the pressure driven displacement of the intima was established for each quadrant and a binary score (significant displacement or no displacement) was decided by consensus of the three analysts.

\section{Statistical analysis}

Continuous variables are presented as mean $\pm \mathrm{SD}$. The sensitivity (proportion of deformable quadrants 
where significant displacement of the intima is present), specificity (proportion of non-deformable quadrants where no displacement of the intima is present), positive predictive value (proportion of quadrants with significant displacement of the intima where deformable tissue is present) and negative predictive value (proportion of quadrants with no displacement of the intima where no deformable tissue is present) of the 3-D ECGgated IVUS to detect deformable regions of the coronaries was evaluated.

\section{Results}

We studied 9 male patients with a mean age $59 \pm 9.3$. The study vessel was the left anterior descending artery in $3(33.3 \%)$, the left circumflex artery in $2(22.2 \%)$ and the right coronary artery in $4(44.4 \%)$ patients.

Thirty-seven frames were selected for paired analysis and 6 were excluded due to significant longitudinal movement of the catheter, which was assessed using longitudinal and cross-sectional views.

As aforementioned, 4 quadrants per frame were individually assessed leading to a total of 124 quadrants.

Overall, the number of highly deformable quadrants was low $(n=7,5.6 \%$ of the total). Conversely, the number of quadrants where significant displacement of the intima was present was slightly higher $(n=18,14.5 \%)$.

The sensitivity, specificity, positive predictive value and negative predictive value of 3-D ECGgated IVUS to detect deformable quadrants as assessed by palpography were $42.9,87.2,16.7$, and $96.2 \%$ respectively.

\section{Discussion}

The sensitivity and specificity of palpography to detect vulnerable plaques has recently been assessed in post-mortem human coronary arteries where vulnerable plaques were detected with a sensitivity of $88 \%$ and a specificity of $89 \%$ [12]. In addition to ex-vivo studies, this technique has also been tested in-vivo, where palpography detected a high incidence of deformable plaques in ACS patients. Furthermore, the number of highly deformable lesions was correlated to the clinical presentation and levels of C-reactive protein [3].

The detection of vulnerable plaques by IVUS is mainly based on a series of case reports [15-18]. These reports describe morphologic features of already ruptured plaques but not the prospective detection of rupture-prone plaques. Nevertheless, one prospective study showed that large eccentric plaques containing an echolucent zone by IVUS were found to be at increased risk of instability even though the lumen area was preserved at the time of initial study [19].

Conventional gray-scale IVUS studies commonly evaluate the static character of the tissue. As the vessel wall is always subject to shear and wall stress, understanding the dynamic characteristics of coronary atherosclerosis by analyzing the intimal displacement velocity in the radial direction could provide an additive value to gray-scale IVUS [11].

In the current report, we evaluated the sensitivity and specificity of 3-D ECG-gated IVUS to detect deformable (high strain) plaques assessed by palpography. The sensitivity was low and the specificity was high. It is noteworthy that only a low percent $(5.6 \%)$ of the analyzed frames presented a high strain. 3-D ECG-gated IVUS seems thus poorly sensitive to detect deformable spots. However, these preliminary findings may suggest that the absence of significant displacement of the intima appear to be highly accurate to predict the absence of underlying deformable tissue.

In coronary arteries, the natural motion of the catheter, related to blood flow pattern during systole and diastole and to the contraction of the heart, is inevitable. During systole, blood flow is low, the heart is contracting, and the catheter moves toward the ostium. Conversely, during the diastolic phase, blood flow increases, the heart relaxes, and the catheter moves distally away from the ostium [20]. It has been reported that ECGgating the IVUS acquisition can significantly reduce the motion artifacts $[8,20]$. However, in the present study all vessels presented some longitudinal movement artifact that could have ultimately influenced the interpretation of the images. 


\section{Limitations}

This study included a small number of patients. Nevertheless, the conductance of large in vivo studies of this type is difficult due to obvious ethical issues. The small prevalence of deformable (high-strain) quadrants could influence the results. Accordingly, interpretation of these results should be cautious and regarded as preliminary. Despite the ECG-gating, motion artifacts seem to be inevitable and could potentially have created misinterpretation of the images. The present is a comparison between quantitative and qualitative techniques. The lack of a quantitative definition of both significant intimal displacement and longitudinal movement could potentially influence the results. However, characterization of such definitions was performed by 3 experienced IVUS analysts. On the other hand, the "gold standard" for sensitivity analysis was an only recently validated technique. However it has shown a high sensitivity and specificity to identify vulnerable plaques [12].

\section{Conclusion}

In this pilot in vivo study, the intimal displacement velocity in the radial direction assessed by grayscale 3-D ECG-gated IVUS failed to correlate with highly deformable regions. The sensitivity was low and the specificity was high. Dynamic assessment of 3-D ECG-gated IVUS seems thus poorly sensitive to detect deformable spots. However, these preliminary findings suggest that the absence of significant displacement of the intima might potentially predict the absence of underlying deformable tissue. Larger studies using a both qualitative and quantitative approach are needed to further investigate the value of these findings.

\section{References}

1. Kannel WB, Doyle JT, McNamara PM, Quickenton P, Gordon T. Precursors of sudden coronary deathFactors related to the incidence of sudden death. Circulation 1975; 51: 606-613.

2. Falk E, Shah PK, Fuster V. Coronary plaque disruption. Circulation 1995; 92: 657-671.

3. Schaar JA, Regar E, Mastik F, et al. Incidence of high-strain patterns in human coronary arteries: assessment with three- dimensional intravascular palpography and correlation with clinical presentation. Circulation 2004; 109: 2716-2719.

4. Regar E, Schaar J, van der Giessen W, van der Steen AF, Serruys PW. Real-time, in-vivo optical coherence tomography of human coronary arteries using a dedicated imaging wire. Am J Cardiol 2002; 90: 129H.

5. Asakura M, Ueda Y, Yamaguchi O, et al. Extensive development of vulnerable plaques as a pan-coronary process in patients with myocardial infarction: an angioscopic study. J Am Coll Cardiol 2001; 37: 1284-1288.

6. Burleigh MC, Briggs AD, Lendon CL, Davies MJ, Born GV, Richardson PD. Collagen types I and III, collagen content, GAGs and mechanical strength of human atherosclerotic plaque caps: span-wise variations. Atherosclerosis 1992; 96: 71-81.

7. Loree HM, Kamm RD, Stringfellow RG, Lee RT. Effects of fibrous cap thickness on peak circumferential stress in model atherosclerotic vessels. Circ Res 1992; 71: 850-858.

8. Bruining N, von Birgelen C, de Feyter PJ, et al. ECG-gated versus nongated three-dimensional intracoronary ultrasound analysis: implications for volumetric measurements. Cathet Cardiovasc Diagn 1998; 43: 254-260.

9. von Birgelen C, de Vrey EA, Mintz GS, et al. ECG-gated three-dimensional intravascular ultrasound: feasibility and reproducibility of the automated analysis of coronary lumen and atherosclerotic plaque dimensions in humans. Circulation 1997; 96: 2944-2952.

10. von Birgelen C, de Feyter PJ, de Vrey EA, et al. Simpson's rule for the volumetric ultrasound assessment of atherosclerotic coronary arteries: a study with ECG-gated threedimensional intravascular ultrasound. Coron Artery Dis 1997; 8: 363-369.

11. Saijo Y, Tanaka A, Owada N, Akino Y, Nitta S. Tissue velocity imaging of coronary artery by rotating-type intravascular ultrasound. Ultrasonics 2004; 42: 753-757.

12. Schaar JA, De Korte CL, Mastik F, et al. Characterizing vulnerable plaque features with intravascular elastography. Circulation 2003; 108: 2636-2641.

13. de Korte CL, van der Steen AF, Cespedes EI, Pasterkamp G. Intravascular ultrasound elastography in human arteries: initial experience in vitro. Ultrasound Med Biol 1998; 24: 401-408.

14. Konofagou E, Ophir J. A new elastographic method for estimation and imaging of lateral displacements, lateral strains, corrected axial strains and Poisson's ratios in tissues. Ultrasound Med Biol 1998; 24: 1183-1199.

15. Ge J, Haude M, Gorge G, Liu F, Erbel R. Silent healing of spontaneous plaque disruption demonstrated by intracoronary ultrasound. Eur Heart J 1995; 16: 1149-1151.

16. Jeremias A, Ge J, Erbel R. New insight into plaque healing after plaque rupture with subsequent thrombus formation detected by intravascular ultrasound. Heart 1997; 77: 293.

17. Ge J, Chirillo F, Schwedtmann J, et al. Screening of ruptured plaques in patients with coronary artery disease by intravascular ultrasound. Heart 1999; 81: 621-627.

18. Rioufol G, Finet G, Ginon I, et al. Multiple atherosclerotic plaque rupture in acute coronary syndrome: a three-vessel 
intravascular ultrasound study. Circulation 2002; 106: 804808.

19. Yamagishi M, Terashima M, Awano K, et al. Morphology of vulnerable coronary plaque: insights from follow-up of patients examined by intravascular ultrasound before an acute coronary syndrome. J Am Coll Cardiol 2000; 35: 106-111.

20. Arbab-Zadeh A, DeMaria AN, Penny WF, Russo RJ, Kimura BJ, Bhargava V. Axial movement of the intravascular ultrasound probe during the cardiac cycle: implications for three-dimensional reconstruction and measurements of coronary dimensions. Am Heart J 1999; 138: $865-872$.

Address for correspondence: Prof. P.W. Serruys, Thoraxcenter, Bd 406, Dr. Molewaterplein 40, 3015-GD Rotterdam,

The Netherlands

Tel.: + 31-10-4635260; Fax: + 31-10-4369154

E-mail: p.w.j.c.serruys@erasmusmc.nl 